\title{
Conditions on Input Disturbance Suppression for Multivariable Nonlinear Systems on the Basis of Feedforward Passivity
}

\author{
Steven W. $\mathrm{Su}^{1}$, Jie $\mathrm{Bao}^{1 *}$ and Peter L. Lee ${ }^{2}$ \\ ${ }^{1}$ School of Chemical Engineering \& Industrial Chemistry \\ The University of New South Wales, \\ UNSW, Sydney, NSW 2052, Australia \\ ${ }^{2}$ Division of Engineering \& Science \\ Curtin University of Technology \\ GPO Box U1987, Perth, WA 6845, Australia
}

\begin{abstract}
In this paper, we consider the problem of input disturbance suppression for nonlinear systems based on feedforward passivity. Firstly, we show that integral control can sufficiently suppress a class of slowly variant (including constant) input disturbance and track constant references provided certain closed-loop stability is achieved. Then, sufficient conditions of integral controllability for nonlinear processes are presented. These conditions are further relaxed by using an input and output transformation.
\end{abstract}

\section{Introduction}

An important objective of control system design is to minimize the effects of external disturbances. The problem of disturbance rejection arises in many industrial fields, such as motion-control, active noise control, vibration control and chemical process control. Slowly variant or constant input disturbance is often encountered in process control.

The problem of constant input disturbance suppression for nonlinear systems has drawn significant attention in academia (e.g., [1], [2] and [6]). It was proved that integral control can achieve asymptotic tracking and asymptotic disturbance rejection for nonlinear multivariable systems, provided that the disturbance is bounded and goes to a constant exponentially ([1] and [2]). Some recent papers, such as [6], dealt with the

\footnotetext{
${ }^{*}$ Author to whom correspondence should be addressed. Email: j.bao@unsw.edu.au. Tel: +61 (2) 9385-6755. Fax: +61 (2)
} 9385-5966. 
robust constant disturbance suppression problem for some special models (with triangular structure) by using integral control with some nonlinear design techniques (e.g., backstepping).

This paper considers the problem of input disturbance suppression for a class of slowly variant disturbance signals which are not necessarily bounded. It is shown that integral control is sufficient to reject such input disturbances provided that the closed loop is exponentially stable. An $L_{2}$ bound on the plant output under the disturbance is also derived. Furthermore, sufficient conditions of integral controllability for nonlinear processes are proposed. This is to answer the question that what conditions a nonlinear process needs to satisfy such that integral action can be employed to achieve offset free control. The proposed conditions are based on the concept of feedforward passivation, and thus can be used for stable nonlinear processes which are non-minimum phase and/or have relative degrees larger than one.

The paper is organised as follows. The problem concerned is described in Section 2. In Section 3, the sufficient condition for slowly variant disturbance suppression is presented. In Section 4, steady state conditions are given for integral controllability. An input and output transformation is then introduced to relax the above condition. Finally in Section 5, we illustrate the proposed method by using an example of mixing plant control.

\section{Problem Description}

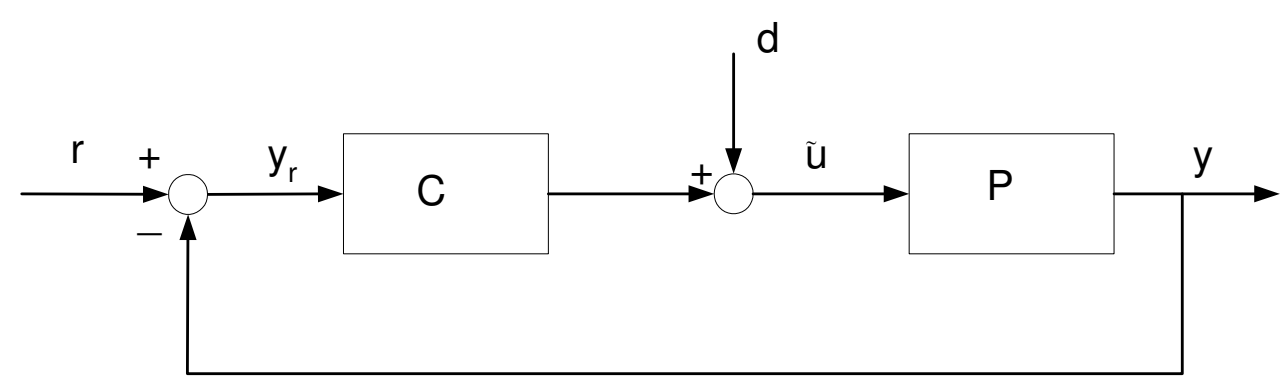

Figure 1: A nonlinear constant disturbance suppression problem

We consider the input disturbance rejection problem for a nonlinear MIMO system, as shown in Figure 1. The feedback system consists of a nonlinear plant $P$ and controller $C$, forced by a constant command signal $r$, as well as an input disturbance $d$. We assume that the nonlinear plant $P$ has the same number of inputs and outputs. Here, $y_{r}$ is the reference tracking error, and $\tilde{u}$ is the input to the plant. We also assume the input disturbance is slowly variant such that its derivative is in $\mathcal{L}_{2}$ space, as defined below:

Definition 1 Signal $d(t)$ is said to be "slowly variant" if $d(t) \in \mathcal{D}_{d \mathcal{L}_{2}}=\left\{v(t): v(t)=\int_{0}^{t} \tilde{v}(\tau) d \tau, \forall \tilde{v}(t) \in \mathcal{L}_{2}\right\}$.

It should be noted that disturbance $d \in \mathcal{D}_{d \mathcal{L}_{2}}$ is more general than the disturbance as considered in [1] and [2], and could be unbounded signal. Consider the case that $\tilde{d}=\frac{1}{1+t} \in \mathcal{L}_{2}, d=\int_{0}^{t} \frac{1}{1+\tau} d \tau$ is unbounded 


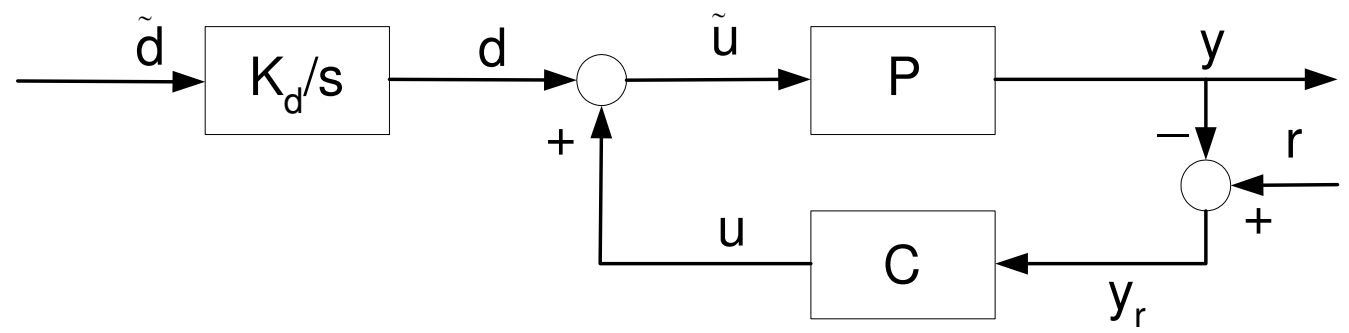

Figure 2: An equivalent block diagram

when $t$ goes to infinity. A class of disturbances encountered in the area of control engineering application can be approximated by the slowly variant signal defined above. For example, system drifting caused by rising temperature can be regarded as a slowly variant disturbance for an amplifier system. The gyroscope's zero drift and accelerometer's zero bias of an inertial navigation system are other examples.

The problem of suppression of the above slowly variant disturbances is illustrated by Figure 2. The gain matrix $K_{d}$ is diagonal and positive definite. Plant $P$ is stable (Later, we shall be more precise concerning the type of stability), and its state space representation is modeled is given below:

$$
P:\left\{\begin{aligned}
\dot{x} & =f(x, \tilde{u}) \\
y & =g(x, \tilde{u}) .
\end{aligned}\right.
$$

If there is no particular declaration in this paper, we suppose that $f: \mathcal{R}^{n} \times \mathcal{R}^{m} \mapsto \mathcal{R}^{n}$ and $g: \mathcal{R}^{n} \times \mathcal{R}^{m} \mapsto \mathcal{R}^{m}$ are unbiased in the sense that

$$
\left\{\begin{array}{l}
f(0,0)=0 \\
g(0,0)=0 .
\end{array}\right.
$$

We assume that the constant reference input signal $r=0$ at this stage.

\section{Sufficient Conditions for slowly variant Disturbance Rejection}

Papers [1] and [2] proved that if a plant can be stabilised by an integral controller and the closed-loop is asymptotically stable (AS), then constant input disturbance rejection and constant reference tracking are guaranteed. In [11] and [12], it was shown that for SISO systems an output feedback $\mathcal{H}_{\infty}$ controller must contain an integrator in the controller to achieve robust input disturbance suppression. In this section, we will prove that for MIMO systems an integration matrix ${ }^{1}$ controller can guarantee the existence of a finite $\mathcal{H}_{\infty}$ norm from disturbance generating signal $\tilde{d}$ to system output $y$ (see Figure 3 ).

In order to set up the relationships between input-output stability [13] and Lyapunov stability for this input disturbance rejection problem, we need to have the following preliminary definitions and theorems:

\footnotetext{
${ }^{1}$ We call a diagonal square matrix (assume the dimension of this matrix is $l$ ) with diagonal elements as $k_{i} / s\left(k_{i}>0\right)$, $i \in\{1,2, \cdots, l\}$ an integration matrix.
} 


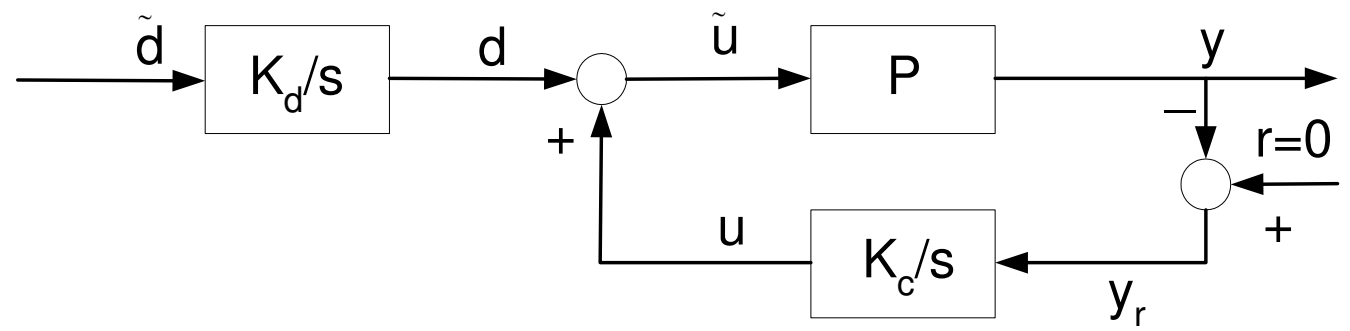

Figure 3: The input-output stability with integration matrix controller

Definition 2 [13] A system is globally exponentially stable (GES) if and only if there exists a Lyapunov function $U(x) \geq 0$ such that

$$
\rho_{1}|x|^{2} \leq U(x) \leq \rho_{2}|x|^{2}
$$

and with zero input

$$
\frac{d}{d t} U(x(t)) \leq-\rho_{3}|x|^{2} .
$$

Where $\rho_{i}>0, i=1,2,3$ are suitable scalar constants and $|\cdot|$ stands for Euclidean norm. If these conditions hold, it follows that there exists some constant $\rho \geq 0$ such that with $x(0)=x_{0}$,

$$
|x(t)| \leq \rho\left|x_{0}\right| e^{-\rho_{3} t / 2}, \quad \text { for all } t \geq 0 .
$$

By local exponential stability (LES) we mean that this definition is valid at least for $x$ in a neighbourhood of $x=0$.

Definition 3 [13] Consider the nonlinear system of the form

$$
\left\{\begin{array}{l}
\dot{x}=f(x, u) \\
y=g(x, u) .
\end{array}\right.
$$

The system (3) is said to be " $\mathcal{L}_{p}$-stable with finite gain" if there exist constants $b_{p}$ and $\gamma_{p}<\infty$ such that $u \in \mathcal{L}_{p}^{m} \Longrightarrow y \in \mathcal{L}_{p}^{l}$ and $\|y\|_{p} \leq \gamma_{p}\|u\|_{p}+b_{p}$. If $p=2, \gamma_{p}$ is said to be the $\mathcal{L}_{2}$ bound from $u$ to $y$.

The system (3) is said to be " $\mathcal{L}_{p}$-stable without bias" if there exists a constant $\gamma_{p}<\infty$ such that $x(0)=0$, $u \in \mathcal{L}_{p}^{m} \Longrightarrow y \in \mathcal{L}_{p}^{l}$ and $\|y\|_{p} \leq \gamma_{p}\|u\|_{p}$.

The system (3) is "small signal $\mathcal{L}_{p}$-stable without bias" if there exist constants $r_{p}>0$ and $\gamma_{p}<\infty$ such that $x(0)=0, u \in \mathcal{L}_{p}^{m}$ with $\|u\|_{p} \leq r_{p} \Longrightarrow y \in \mathcal{L}_{p}^{l}$ and $\|y\|_{p} \leq \gamma_{p}\|u\|_{p}$.

Theorem 4 [13] Consider the system described by equation (3). Suppose that $f: \mathcal{R}^{n} \times \mathcal{R}^{m} \mapsto \mathcal{R}^{n}$ and $g: \mathcal{R}^{n} \times \mathcal{R}^{m} \mapsto \mathcal{R}^{l}$ are unbiased in the sense that

$$
\left\{\begin{array}{l}
f(0,0)=0 \\
g(0,0)=0 .
\end{array}\right.
$$


which ensures that $x=0$ is an equilibrium of the unforced system

$$
\dot{x}=f(x, 0) .
$$

Suppose that $x=0$ is an exponentially stable equilibrium of (5), and that $f$ is $C^{1}$. Suppose also that $f$ and $g$ are locally Lipschitz continuous at $(0,0)$, that is, suppose there exist finite constants $k_{f}, k_{g}, r$ such that

$$
\begin{aligned}
& \|f(x, u)-f(z, v)\|_{2} \leq k_{f}\left[\|x-z\|_{2}+\|u-v\|_{2}\right], \forall(x, u)(z, v) \in B_{r}, \\
& \|g(x, u)-g(z, v)\|_{2} \leq k_{g}\left[\|x-z\|_{2}+\|u-v\|_{2}\right], \forall(x, u)(z, v) \in B_{r} .
\end{aligned}
$$

Here, $B_{r}$ is the open ball of the radius $r$, that is, $B_{r}=\left\{x:\left\|x-x_{0}\right\|<r\right\}$. Then the system (3) is small signal $\mathcal{L}_{p}$-stable without bias for each $p \in[1, \infty)$. If $x=0$ is a globally exponentially stable equilibrium,

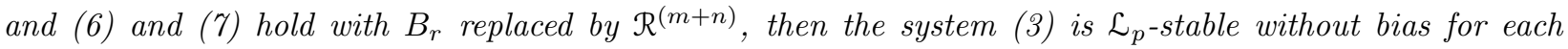
$p \in[1, \infty)$. Furthermore, there exists a Lyapunov function $U(x) \geq 0$ which satisfies the requirements of exponential stability of Definition 2, and the gain $\gamma_{p}$ is related to the constants $\rho_{i}$ defining the properties of $U(x)$ by

$$
\|y\|_{p} \leq k_{g}\left[\frac{\rho_{3} k_{f}}{4 \rho_{1}^{2} \rho_{2}^{2}}+1\right]\|u\|_{p}
$$

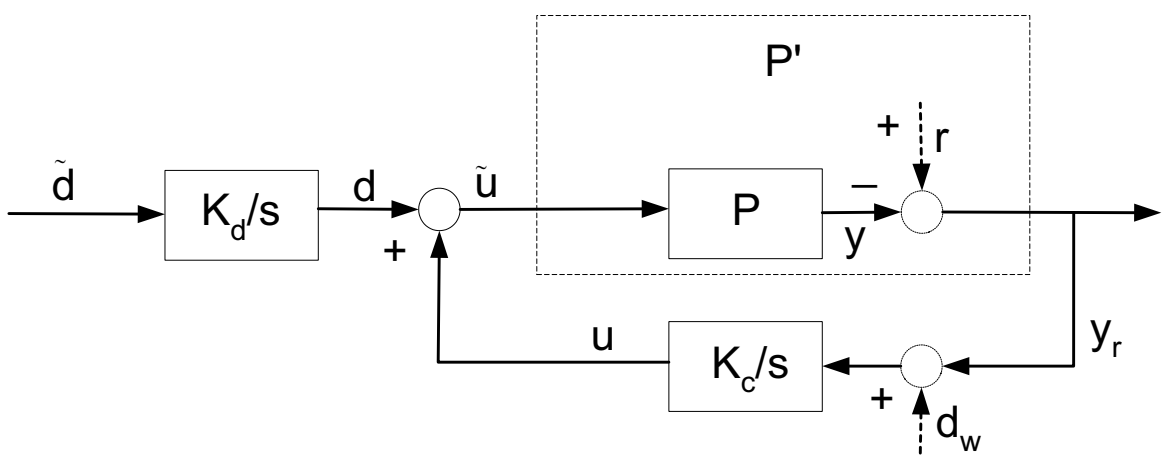

Figure 4: Nonlinear input disturbance suppression

Theorem 5 Consider the system depicted in Figure 4. Plant P is described by equations (1) and (2). Suppose that $(0,0)$ is an exponentially stable equilibrium of the unforced closed loop $\left(P, \frac{K_{c}}{s}\right)$. Further, assume that $f$ is $C^{1}$, and that $f, g$ are locally Lipschitz continuous at $(0,0)$ with Lipschitz constants $k_{f}$ and $k_{g}$ to the Euclidean norm $\|\cdot\|_{2}$ (See Definition 2 and Theorem 4.).

Then the system depicted in Figure 4 is small signal $\mathcal{L}_{2}$ stable without bias from $\tilde{d}$ to $y$.

If $(0,0)$ is a globally exponentially stable equilibrium, and $f, g$ are globally Lipschitz continuous at $(0,0)$, then the system is $\mathcal{L}_{2}$ stable without bias. 


\section{Proof}

Consider Figure 4. Suppose that there is an additional input $d_{w}$ to the integration matrix controller $\frac{K_{c}}{s}$. Define the $\mathcal{H}_{\infty}$ norms of the systems from $\tilde{d}$ to $y$ and from $d_{w}$ to $y$ are $\gamma_{\tilde{d} y}$ and $\gamma_{d_{w} y}$ respectively. If we set

$$
d_{w}=K_{d} K_{c}^{-1} \tilde{d}
$$

then the input $d_{w}$ is equivalent to the input of the signal $\tilde{d}$. That is, we can replace the disturbance input $\tilde{d} \in \mathcal{L}_{2}$ of the system depicted in Figure 4 by the equivalent signal $d_{w} \in \mathcal{L}_{2}$. Because $(0,0)$ is an exponentially stable equilibrium of the unforced closed loop $\left(P, \frac{K_{c}}{s}\right)$, then we will see that, according to Theorem 4 , a finite gain $\gamma_{d_{w} y}$ from $d_{w}$ to $y$ exists. Then, from equation (8), we conclude that a finite gain $\gamma_{\tilde{d} y}$ from $\tilde{d}$ to $y$ also exists.

More precisely, the augmented system with input $d_{w}$ and output $y$ can be described as equation (9).

$$
\left\{\begin{aligned}
\dot{x} & =f(x, \xi) \\
\dot{\xi} & =K_{c}\left(-g(x, \xi)+d_{w}\right) \\
y & =g(x, \xi) .
\end{aligned}\right.
$$

It can be verified that the state space equations are exactly the same by using either signal $d_{w}$ or $\tilde{d}$ as a disturbance generating input provided $d_{w}=K_{d} K_{c}{ }^{-1} \tilde{d}$.

Let $x_{a}=\left[\begin{array}{ll}x^{T} & \xi^{T}\end{array}\right]^{T}$, then the above equation can be rewritten in the form:

$$
\left\{\begin{array}{l}
\dot{x}_{a}=f_{a}\left(x_{a}, d_{w}\right) \\
y=g_{a}\left(x_{a}\right)
\end{array}\right.
$$

Here, $f_{a}\left(x_{a}, d_{w}\right)=\left[\begin{array}{c}f\left(x_{a}\right) \\ K_{c}\left(-g\left(x_{a}\right)+d_{w}\right)\end{array}\right], g_{a}\left(x_{a}\right)=g(x, \xi)$.

Then, $\forall\left(x_{a}, d_{w}\right),\left(x_{a}^{\prime}, d_{w}^{\prime}\right) \in \mathcal{R}^{(n+2 l)}$,

$$
\begin{aligned}
& \left\|f_{a}\left(x_{a}, d_{w}\right)-f_{a}\left(x_{a}^{\prime}, d_{w}^{\prime}\right)\right\|_{2} \\
= & \| \begin{array}{c}
f\left(x_{a}\right)-f\left(x_{a}^{\prime}\right) \\
K_{c}\left(-g\left(x_{a}\right)+g\left(x_{a}^{\prime}\right)\right)+K_{c}\left(d_{w}-d_{w}^{\prime}\right)
\end{array} \\
\leq & k_{f}\left(\left\|x-x^{\prime}\right\|_{2}+\left\|\xi-\xi^{\prime}\right\|_{2}\right)+k_{g}\left(\left\|x-x^{\prime}\right\|_{2}+\left\|\xi-\xi^{\prime}\right\|_{2}\right)+\left\|K_{c}\right\|_{2}\left\|d_{w}-d_{w}^{\prime}\right\|_{2} \\
\leq & \sqrt{2}\left(k_{f}+k_{g}\right)\left\|x_{a}-x_{a}^{\prime}\right\|_{2}+\left\|K_{c}\right\|_{2}\left\|d_{w}-d_{w}^{\prime}\right\|_{2} \\
\leq & k_{f_{a}}\left(\left\|x_{a}-x_{a}^{\prime}\right\|_{2}+\left\|d_{w}-d_{w}^{\prime}\right\|_{2}\right) .
\end{aligned}
$$

Here, $\left\|K_{c}\right\|_{2}=\sqrt{\lambda_{\max }\left(K_{c}^{T} K_{c}\right)}$, and $k_{f_{a}}=\max \left\{\sqrt{2}\left(k_{f}+k_{g}\right),\left\|K_{c}\right\|_{2}\right\}$.

Similarly, it is obvious that $\left\|g_{a}\left(x_{a}\right)-g_{a}\left(x_{a}^{\prime}\right)\right\|_{2} \leq k_{g_{a}}\left\|x_{a}-x_{a}^{\prime}\right\|_{2}$, where $k_{g_{a}}=k_{g}$.

In view of the assumption that $(0,0)$ is an exponentially stable equilibrium of the unforced closed loop $\left(P, \frac{K_{c}}{s}\right)$, there exists a Lyapunov function $U(x) \geq 0$, which satisfies the requirements of Definition 2. According to Theorem 4 the finite gain $\gamma_{d_{w} z}$ from $d_{w}$ to $y$ is $\gamma_{d_{w} z}=\left(\frac{\rho_{3} k_{f a}}{4 \rho_{1}{ }^{2} \rho_{2}{ }^{2}}+1\right)$, where the constants $\rho_{i}$ are defined 
by the properties of $U(x)$. Therefore, $\gamma_{\tilde{d} y} \leq\left\|K_{d}\right\|_{2}\left\|K_{c}{ }^{-1}\right\|_{2}\left(\frac{\rho_{3} k_{f a}}{4 \rho_{1}^{2} \rho_{2}{ }^{2}}+1\right)$.

The significance of Theorem 5 is that it shows that if a controller is augmented with an integration matrix, and the closed loop is exponentially stable, then input-output stability from $\tilde{d}$ to $y$ is ensured. Note that there is an integral weighting function between $\tilde{d}$ and $d$ which ensures that for a "slowly variant" disturbance $d$ (see Definition 1 ), the output signal $y$ is in $\mathcal{L}_{2}$ and hence asymptotically goes to zero. This implies that integral control is sufficient to suppress the "slowly variant" input disturbance which includes asymptotically constant disturbances.

Note 6 Consider plant $P^{\prime}$ in Figure 4. If we have a nonzero constant reference input $r$, we can consider the original plant $P$ and reference input $r$ to be equivalent to a new plant $P^{\prime}$ with an equilibrium point $\left(x_{e}\right)$, where $g\left(x_{e}, u_{r}\right)=r$. Sufficient conditions for stability in this situation are that the conditions of Theorem 5 are satisfied for the new equilibrium point (any equilibrium $x_{e}$ under investigation can be translated to the origin by redefining the state $x$ as $\left.x-x_{e}[10]\right)$.

\section{Sufficient Conditions for Process Integral Controllability}

In this section, sufficient conditions of integral controllability for nonlinear processes are proposed based on feedforward passivation. The concept of passivity and the Passivity Theorem for nonlinear system are introduced first.

Definition 7 [10] Consider a nonlinear system P:

$$
\left\{\begin{array}{l}
\dot{x}=f(x, u) \quad x \in \mathcal{R}^{n} \\
y=h(x, u), \quad u, y \in \mathcal{R}^{m}
\end{array}\right.
$$

and assume that the state $x(t)$, as a function of time, is uniquely determined by its initial value $x(0)$ and the input function $u(t)$. Suppose that the above system has an equilibrium at the origin, that is, $f(0,0)=0$, and $h(0,0)=0$.

Assume that associated with the system $P$ is a function $w: \mathcal{R}^{m} \times \mathcal{R}^{m} \mapsto \mathcal{R}$, called the supply rate, which is locally integrable for every $u \in \mathcal{U}$. Let $\mathcal{X}$ be a connected subset of $\mathcal{R}^{n}$ containing the origin. If there exists a function $S: \mathcal{X} \mapsto \mathcal{R}^{+}$(denote $\mathcal{R}^{+}=[0, \infty)$ ), $S(0)=0$, such that for all $x \in X$ :

$$
S(x(T))-S(x(0)) \leq \int_{0}^{T} s(u(t), y(t)) d t
$$

for all $u \in \mathcal{U}$ and all $T \geq 0$ such that $x(t) \in X$ for all $t \in[0, T]$, then we say that system $P$ is dissipative in $X$ with the supply rate $w(u, y)$. The function $S(x)$ is then called a storage function. 
System $P$ is said to be passive, Input Feedforward Passive $(\operatorname{IFP}(\nu))$ or Nonlinear Input Feedforward Passive (NIFP), if it is dissipative with supply rate

$$
\begin{gathered}
w(u, y)=u^{T} y, \\
w(u, y)=u^{T} y-\nu u^{T} u
\end{gathered}
$$

or

$$
w(u, y)=u^{T} y-\nu^{T}(u) u
$$

respectively. Where $\nu(u)=\left[\nu_{1}\left(u_{1}\right), \cdots, \nu_{m}\left(u_{m}\right)\right]^{T}$, and $\nu_{i}\left(u_{i}\right)$ are in the sector $(0,+\infty)$ or $(0,-\infty), i=$ $1,2, \cdots, m$.

On the basis of the concept of passive systems, the stability of two interconnected nonlinear systems can be determined by the following Passivity Theorem:

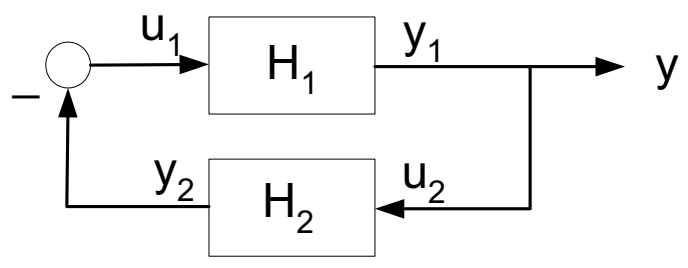

Figure 5: Feedback interconnections of two systems.

Theorem 8 [10] Assume that the systems $H_{1}$ and $H_{2}$ are dissipative with respect to the following supply rates:

$$
w_{i}\left(u_{i}, y_{i}\right)=u_{i}^{T} y_{i}^{T}-\rho_{i}^{T}\left(y_{i}\right) y_{i}-\nu_{i}^{T}\left(u_{i}\right)\left(u_{i}\right), i=1,2
$$

Furthermore assume that they are zero-state detectable (ZSD) [10] and that their respective storage functions $S_{1}\left(x_{1}\right)$ and $S_{2}\left(x_{2}\right)$ are $C^{1}$. Then the equilibrium $\left(x_{1}, x_{2}\right)=(0,0)$ of the feedback interconnection in Figure 5 is:

$$
\begin{aligned}
& \text { i)stable, if } \nu_{1}^{T}(v) v+\rho_{2}^{T}(v) v \geq 0 \text { and } \nu_{2}^{T}(v) v+\rho_{1}^{T}(v) v \geq 0 . \\
& \text { ii)asymptotically stable, if } \nu_{1}^{T}(v) v+\rho_{2}^{T}(v) v>0 \text { and } \nu_{2}^{T}(v) v+\rho_{1}^{T}(v) v>0 \text {. }
\end{aligned}
$$

Theorem 8 implies the well known result that a feedback system comprised of a passive system and a strictly passive system is asymptotically stable. Therefore, if a process is strictly passive, then it is integral controllable. For non-passive processes, a feedforward subsystem $P_{f f}$ (see Figure 6) can be constructed to passify the process. It should be noted that the final controller (see Figure 6 ) is $K_{c} / s$ with the negative feedback of $P_{f f}$. The conditions under which the subsystem $P_{f f}$ can achieve both input disturbance suppression and reference tracking is investigated. In order to clarify our discussions, we introduce some notations and concepts first. 


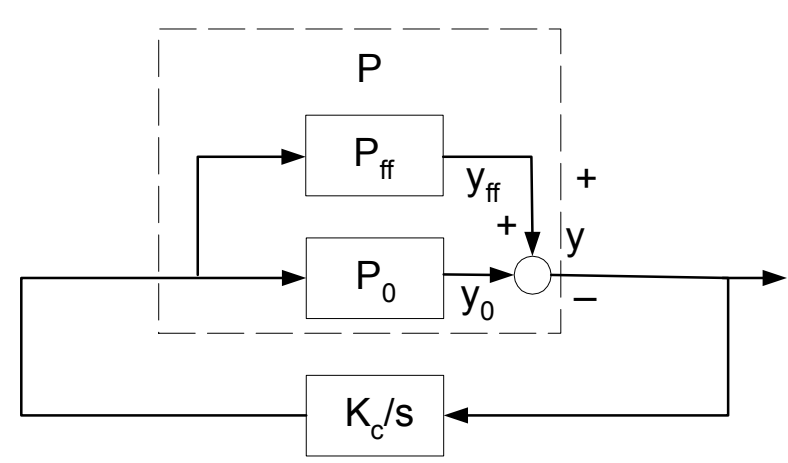

Figure 6: Feedforward passivity

Definition 9 [3] (Nonlinear operator) A nonlinear operator is the operator $M$, which maps u to $y(=M u)$ through the relations

$$
\begin{cases}\dot{x}=f(x, u), \quad x(0)=x_{0} \in \mathcal{R}^{n} \\ y=g(x, u), \quad u, y \in \mathcal{R}^{m}\end{cases}
$$

where the $f$ and $g$ are real analytic vector valued functions.

The nonlinear operator $M$ defined in Definition 9 is "square" in the sense that it has the same number of inputs and outputs.

Definition 10 [3] (Steady state operator) Let $M$ be an input-output stable operator in the domain of $M$ (denoted $D_{0}(M)=\mathfrak{U}_{M}$ ) [14], and $u \in \mathcal{U}_{M}$ with $\lim _{t \rightarrow \infty} u(t)=u_{\infty}<\infty$. Letting $y_{\infty}=\lim _{t \rightarrow \infty} M(u(t)$ ) $\left(y_{\infty}<\infty\right.$ because of the stability assumption), the steady state operator $M_{\infty}$ is defined by

$$
y_{\infty}=M_{\infty}\left(u_{\infty}\right)
$$

While $M$ generally maps function spaces into function spaces, $M_{\infty}(\cdot)$ is mapping vectors from $\mathcal{R}^{m}$ into $\mathcal{R}^{m}$, being a static function.

Consider the operator in (18) where $M$ is assumed input-output stable. For this nonlinear operator, $M_{\infty}$ is given by the system of algebraic equations

$$
\begin{cases}0 & =f\left(x, u_{\infty}\right), \\ y_{\infty} & =g\left(x, u_{\infty}\right) .\end{cases}
$$

Lyapunov stability follows from the input-output stability provided some detectability conditions are met [9]. In order to simplify our discussion, when we say a nonlinear system is an input-output stable, it is assumed that the system also satisfies some detectability conditions to ensure Lyapunov stability around a corresponding equilibrium point.

Definition 11 Assume a nonlinear system $P$ is an input-output stable nonlinear operator. If for any input $u \in \mathcal{U}_{p}$ with $\lim _{t \rightarrow \infty} u(t)=u_{\infty}<\infty$

$$
y_{\infty}=P_{\infty}\left(u_{\infty}\right)=0,
$$

then, we say system $P$ has zeros at steady state. 
Theorem 12 Consider the system depicted in Figure 6. Plant $P$ is described by equations (1) and (2), and is the sum of two subsystems $P_{0}$ and $P_{f f}$ (the feedforward connection or parallel interconnection [10] of the two subsystems). Suppose that $(0,0)$ is an exponentially stable equilibrium of the unforced closed loop $\left(P, \frac{K_{c}}{s}\right)$. Further assume that the subsystem $P_{f f}$ has zeros at steady state, i.e.

$$
y_{f f \infty}=P_{f f \infty}\left(u_{\infty}\right)=0
$$

Then the output of plant $P_{0}$ (i.e., $\left.y_{0}\right)$ will asymptotically go to zero. That is

$$
y_{0 \infty}=\lim _{t \rightarrow \infty} y_{0}(t)=0 .
$$

Proof Since the unforced closed-loop $\left(P, \frac{K_{c}}{s}\right)$ is exponentially stable around $(0,0)$, according to Theorem 5 , the output of plant $P$ (i.e., $y$ ) is in $\mathcal{L}_{2}$ under the slowly variant disturbances $d$. That is,

$$
\lim _{t \rightarrow \infty} y(t)=0 .
$$

From equation (22) and (24), we have that

$$
\lim _{t \rightarrow \infty} y_{0}(t)=\lim _{t \rightarrow \infty} y(t)-\lim _{t \rightarrow \infty} y_{f f}(t)=0
$$

Next, the steady state conditions are presented for a process can be rendered passive by feedforward a subsystem which has zeros at steady state.

Definition 13 Assume a nonlinear system $P$ is an input-output stable operator. We say system $P$ is passive, Input Feedforward Passive or Nonlinear Input Feedforward Passive at steady state, if its steady state input and output relationship $P_{\infty}(\cdot)$ satisfies

$$
\begin{gathered}
u^{T} P_{\infty}(u) \geq 0, \\
u^{T} P_{\infty}(u) \geq \nu u^{T} u,
\end{gathered}
$$

or

$$
u^{T} P_{\infty}(u) \geq \nu^{T}(u) u
$$

respectively.

Theorem 14 For a nonlinear input-output stable operator $P_{0}$, there exists an input output stable feedforward subsystem $P_{f f}$ which has zeros at steady state to passify $P_{0}$ (i.e. $P=P_{0}+P_{f f}$ is passive), if and only if plant $P_{0}$ is passive at steady state. 
Proof (Necessity) For the input-output stable operator $P_{0}$, all feedforward subsystems $P_{f f}$ that render

$$
P=P_{0}+P_{f f}
$$

passive can be parameterized below

$$
P_{f f}=P_{p}-P_{0}
$$

with any passive system $P_{p}$.

Because the subsystem $P_{f f}$ has zeros at steady state, then from equation (29), we conclude that $P_{p}$ and $P_{0}$ have the same input and output functions at steady state, that is, $P_{p \infty}(u)=P_{0 \infty}(u)$. Furthermore, considering that the system $P_{p}$ is passive (at steady state), we conclude that the system $P_{0}$ is passive at steady state.

(Sufficiency) If $P_{0}$ is passive at steady state, then we construct

$$
P_{f f}=P_{0 \infty}(u)-P_{0},
$$

It can be seen that $P=P_{0}+P_{f f}=P_{0 \infty}(u)$ is passive and $P_{f f}$ has zeros at steady state because the steady state operator $P_{f f \infty}=P_{0 \infty}(u)-P_{0 \infty}(u)=0$.

It can be easily verified that replacing the word "passive" by "IFP" or "NIFP", Theorem 14 is still correct.

Note 15 If the subsystem $P_{f f}$ is constructed by using equation (30) Theorem 14, then the passified system $P=P_{0}+P_{f f}$ will be passive, NIFP or IFP $(\nu)$ given that $P_{f f \infty}$ is passive, NIFP or IFP $(\nu)$, respectively. According to the Passivity Theorem (Theorem 8), the closed loop system is AS if a diagonal PI matrix ${ }^{2}$ controller, which is $\operatorname{IFP}\left(\underline{k}_{p}\right)\left(\underline{k}_{p}=\min _{1 \leq i \leq m}\left\{k_{p_{i}}\right\}>0\right)$ is used provided that $P_{f f \infty}$ is NIFP. The stability condition is required to achieve constant disturbance rejection and reference tracking (as in [1] and [2]).

As discussed in Section 3, if an integral controller can exponentially stabilise a nonlinear system then the suppression of the "slowly variant" input disturbance $d \in \mathcal{D}_{d \mathcal{L}_{2}}$ is achievable. A sufficient condition to obtain exponential stability is that the serial connection of $\frac{K_{c}}{s}$ and $P_{0}$ is so called $C^{r}$-semiglobally output feedback exponentially passive $(r \geq 1)$ [4]. Details of the $C^{r}$-semiglobally output feedback exponential passivity $(r \geq 1)$ can be found in [4].

In Theorem 14, we presented an equivalent condition for a plant $P_{0}$ to be made passive $(\operatorname{IFP}(\nu)$ or NIFP) by using a feedforward subsystem which has zeros at steady state. That is, plant $P_{0}$ should be passive $(\operatorname{IFP}(\nu)$ or $N I F P)$ at steady state. For those processes which are not passive at steady state, it is possible to passify them at steady state by using an input transformation

$$
\tilde{u}=\phi(u)
$$

\footnotetext{
${ }^{2}$ We call a diagonal square matrix (assume the dimension of this matrix is $m$ ) with diagonal elements as $k_{p_{i}}+k_{i_{i}} / s\left(k_{p_{i}}>0\right.$, $\left.k_{i_{i}}>0\right), i \in\{1,2, \cdots, m\}$ a PI (Proportion and Integration) matrix.
} 
and an output transformation

$$
\tilde{y}=\psi(y)
$$

to plant $P_{0}$. The functions $\phi(\cdot)$ and $\psi(\cdot)$ are both local diffeomorphism in $\mathcal{U} \in \mathcal{R}^{m}$ [5] [8]. If we assume plant $P_{0}$ is described by

$$
P_{0}:\left\{\begin{aligned}
\dot{x} & =f(x)+g(x) u \\
y & =h(x) .
\end{aligned}\right.
$$

Then, we just need functions $\phi(\cdot)$ and $\psi(\cdot)$ to satisfy

$$
\left\{\begin{array}{l}
0=f(x)+g(x) \phi^{-1}(\tilde{u}) \\
\tilde{y}=\psi(h(x)) .
\end{array}\right.
$$

and

$$
\tilde{y}^{T} \tilde{u} \geq 0 .
$$

If the static input output function of system $P_{0}: y=P_{0 \infty}(u)$ is a local diffeomorphism in $\mathcal{U} \in \mathcal{R}^{m}$, then we can directly select input transformation as $\phi(\cdot)=P_{0 \infty}(\cdot)(\psi(\cdot)=I)$ to transfer the system $P_{0}$ passive at steady state. However, even in this case, both the input and output transformation may be used together to make them more effective and physically meaningful.

\section{Illustrative Example}

In this section, we illustrate the proposed conditions using an example of mixing tank process ([7]) as shown in Figure 7. The tank is fed with two inlet flows with flowrates $F_{1}(t)$ and $F_{2}(t)$. Both inlet flows contain one dissolved material with concentrations $c_{1}$ and $c_{2}$ respectively. The flowrate of the outlet flow is $F(t)$. Assume that the tank is well stirred so that the concentration of the outlet flow is the same as the concentration in

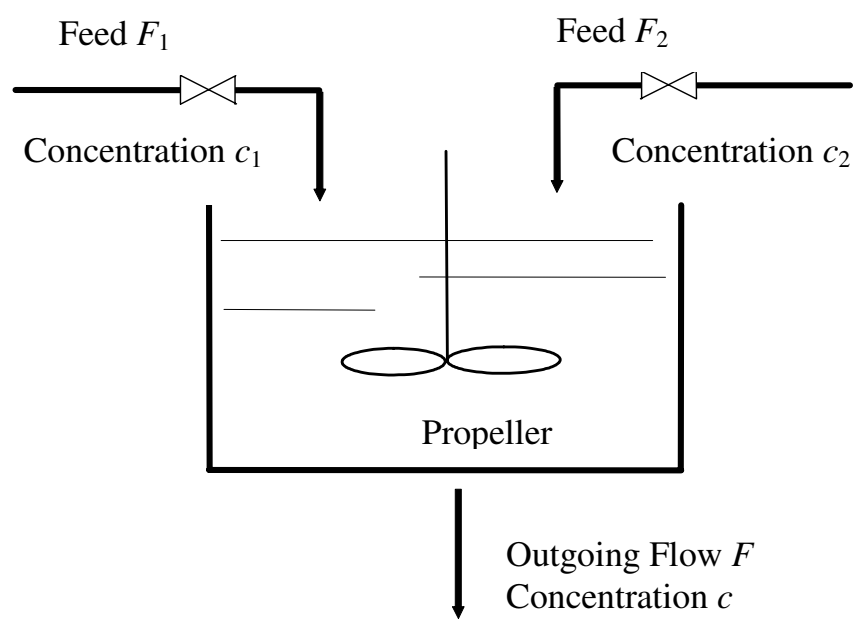

Figure 7: The mixing system. 
the tank. The inlet flowrates, $F_{1}(t)$ and $F_{2}(t)$, are manipulated to control both flowrate $F(t)$ and the outlet concentration $c(t)$ to the desired values under the constant input disturbance. $V(t)$ is the volume of the liquid in the tank.

The process is modelled as follows:

$$
\begin{aligned}
& P_{0}:\left\{\begin{array}{l}
\dot{x}_{1}=-k \sqrt{\frac{x_{1}}{S}}+u_{1}+u_{2} \\
\dot{x}_{2}=-\frac{\left(u_{1}+u_{2}\right) x_{2}}{x_{1}}+\frac{\left(c_{1} u_{1}+c_{2} u_{2}\right)}{x_{1}} .
\end{array}\right. \\
& \left\{\begin{array}{l}
y_{1}=k \sqrt{\frac{x_{1}}{S}} \\
y_{2}=x_{2} .
\end{array}\right.
\end{aligned}
$$

where $x_{1}(t)=V(t), x_{2}(t)=c(t) ; u_{1}(t)=F_{1}(t), u_{2}(t)=F_{2}(t) ; y_{1}(t)=F(t)$ and $y_{2}(t)=c(t)$. Constant $S$ is the cross-sectional area of the tank and $k$ is the discharge coefficient of the exit flowrate. Their values are $S=10 m^{2}$ and $k=5.916$.

The steady state input output function is

$$
\left\{\begin{array}{l}
y_{1}=u_{1}+u_{2} \\
y_{2}=\frac{c_{1} u_{1}+c_{2} u_{2}}{u_{1}+u_{2}},
\end{array}\right.
$$

from which it can be seen that this mixing system is not passive at steady state.

By introducing the following input transformation

$$
\left\{\begin{array}{l}
\hat{u}_{1}=u_{1}+u_{2} \\
\hat{u}_{2}=c_{1} u_{1}+c_{2} u_{2},
\end{array}\right.
$$

and the output transformation

$$
\left\{\begin{array}{l}
\hat{y}_{1}=y_{1} \\
\hat{y}_{2}=y_{1} y_{2},
\end{array}\right.
$$

The original process is transformed into the following system:

$$
\begin{gathered}
P_{0}^{\prime}:\left\{\begin{array}{l}
\dot{x}_{1}=-k \sqrt{\frac{x_{1}}{S}}+\hat{u}_{1} \\
\dot{x}_{2}=-\frac{x_{2} \hat{u}_{1}}{x_{1}}+\frac{\hat{u}_{2}}{x_{1}} .
\end{array}\right. \\
\left\{\begin{array}{l}
\hat{y}_{1}=k \sqrt{\frac{x_{1}}{S}} \\
\hat{y}_{2}=k x_{2} \sqrt{\frac{x_{1}}{S}},
\end{array}\right.
\end{gathered}
$$

with steady state input output function

$$
\left\{\begin{array}{l}
\hat{y}_{1}=\hat{u}_{1} \\
\hat{y}_{2}=\hat{u}_{2} .
\end{array}\right.
$$

It can be seen that the transformed system is $\operatorname{IFP}(1)$ at steady state ${ }^{3}$. Therefore, there exists a feedforward subsystem $P_{f f}$ which has zeros at steady state and renders the mixing system passive, according to Theorem 14. One possible choice is $P_{f f}=P_{0 \infty}-P_{0}^{\prime}=I-P_{0}^{\prime}$.

\footnotetext{
${ }^{3}$ It is noted that although the steady state input output relation of the transformed system is linear, the dynamic part of the system is still nonlinear.
} 


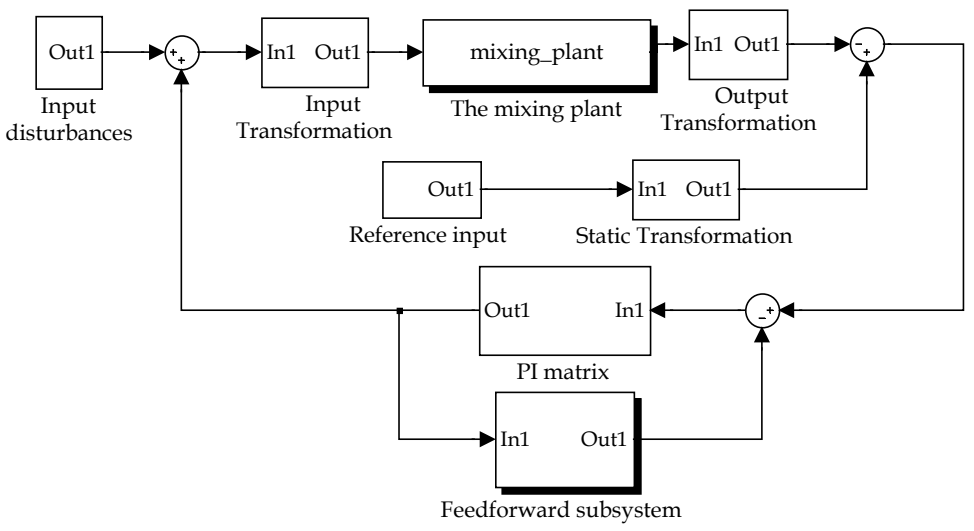

Figure 8: The simulation construction implemented by using Simulink.

System stability under such a controller is tested using Simulink (a product of The MathWorks Inc.). The control diagram is shown in Figure 8. The initial state of the process $P_{0}$ is $x(0)=\left[\begin{array}{ll}0.6 & 1.3\end{array}\right]^{T}$. The reference input is a constant vector $[1,1.6]^{T}$. The input disturbance is $[0.3,-0.3]^{T}$.

During the simulation, we select the diagonal PI matrix with different proportional and integral gains(see figure 8). The simulation results (see Figure 9) show that the closed loop system is stable even with large proportional and integral gains. The input disturbances can be rejected rapidly, and the controller can ensure offset free tracking of the flowrate and the product concentration.

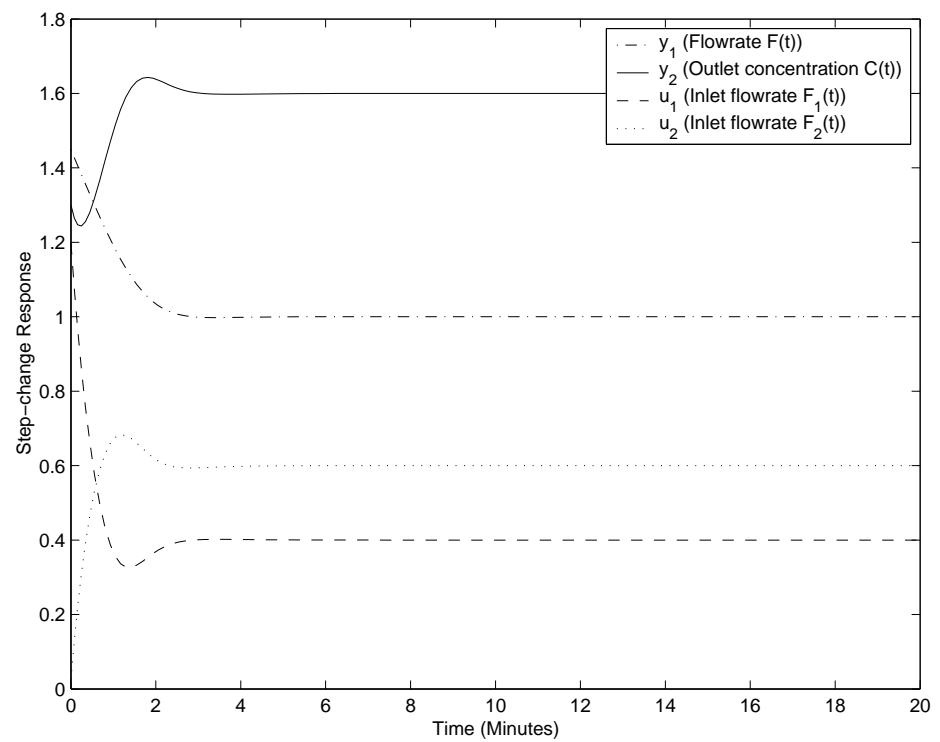

Figure 9: The inputs and outputs of the plant 


\section{Conclusions}

Based on feedforward passivation, this paper considers an input disturbance rejection problem for nonlinear systems. Steady state conditions are given for a system which can be rendered passive by a feedforward subsystem with zeros at steady state. It is shown that plants which satisfy the proposed conditions can effectively suppress constant and slowly variant input disturbances under integral control.

\section{Acknowledgments}

The authors gratefully acknowledge the financial support of the Australian Research Council (Grant A00104473).

\section{References}

[1] C. Desoer and Y. Wang. Foundations of feedback theory for nonlinear dynamical systems. IEEE Trans. on Circuits and Systems, 27(2):104-123, 1980.

[2] C. Desoer and Y.T. Wang. The robust nonlinear servomechanism problem. Int. J. Contr., 29(5):803-828, 1979.

[3] C.G. Economou and M. Morari. Internal model control. 5. extension to nonlinear systems. Ind. Eng. Process Des. Dev., 25:403-411, 1986.

[4] A.L. Fradkov and D.J. Hill. Exponential feedback passivity and stabilizability of nonlinear systems. Automatica, 34:697-703, 1997.

[5] A. Isidori. Nonlinear Control Systems. Springer-Verlag, New York, 1989.

[6] Z.P. Jiang and I. Mareels. Robust nonlinear integral control by partial-state and output feedback. Proceedings of the IEEE Conference on Decision and Control, pages 2084-2089, 2000.

[7] H. Kwakanaak and R. Sivan. Linear Optimal Control Systems. Wiley Interscience, 1972.

[8] R. Marino and P. Tomei. Nonlinear Control Design: Geometric, Adaptive and Robust. Prentice Hall, London, 1995.

[9] R. Ortega, A. Loria, P.J. Nicklasson, and H. Sira-Ramirez. Passivity-based Control of Euler-Lagrange Systems. Springer, London, 1998.

[10] R. Sepulchre, M. Jankovic, and P. Kokotovic. Constructive Nonlinear Control. Springer Verlag, New York, 1996. 
[11] S.W. Su, B.D.O. Anderson, and T.S. Brinsmead. Robust disturbance suppression for nonlinear systems based on $\mathcal{H}_{\infty}$ control. Proceedings of the 39th Conference on Decision and Control, pages 3013-3018, 2000.

[12] S.W. Su, B.D.O. Anderson, and T.S. Brinsmead. Use of integrator in nonlinear $\mathcal{H}_{\infty}$ design for disturbance rejection. Automatica, 38:1951-1957, 2002.

[13] M. Vidyasagar. Nonlinear Systems Analysis. Prentice Hall, New Jersey, 1993.

[14] G. Zames. On the input-output stability of time-varying nonlinear feedback systems part one: Conditions derived using concepts of loop gain, conicity, and positivity. IEEE Trans. on Automatic Control, 11:228238,1966 . 